\title{
Coffee consumption is positively related to insulin secretion in the Shanghai High-Risk Diabetic Screen (SHiDS) Study
}

\author{
Fei Gao ${ }^{1 \dagger}$, Yinan Zhang ${ }^{2 \dagger}$, Sheng Ge ${ }^{3}$, Huijuan Lư ${ }^{4}$, Ruihua Chen ${ }^{4}$, Pingyan Fang ${ }^{4}$, Yixie Shen ${ }^{4}$, \\ Congrong Wang ${ }^{4^{*}}$ (D) and Weiping $\mathrm{Jia}^{4^{*}}$
}

\begin{abstract}
Background: It has been proved that coffee consumption was associated with a lower risk of type 2 diabetes mellitus. But the benefit effect of coffee on hyperglycemia in Chinese population was largely unknown. Besides, the relationship of coffee intake and diabetic pathogenesis was still unclear.

Methods: The study population was selected from the Shanghai High-Risk Diabetic Screen (SHiDS) project. A total of 1328 individuals over 18 years of age who have the information of coffee intake were enrolled in the study from 2012 to 2016. Each participant finished a five-point $75 \mathrm{~g}$ oral glucose tolerance test and finished a standard questionnaire. Insulin resistance was evaluated by HOMA-IR and insulin secretion was evaluated by HOMA- $\beta$, Stumvoll first phase and second phase indexes.

Results: Coffee consumption group had lower plasma glucose levels at 2-h and 3-h and higher insulin levels at fasting, 30-min and 1-h during OGTT after adjustment with age, fat\%, BMI, waist, tea intake, smoking habit, alcohol intake, diabetes family history and educational status ( $\mathrm{P}$ for $\mathrm{PG} 2 \mathrm{~h}=0.002$; $\mathrm{P}$ for $\mathrm{PG} 3 \mathrm{~h}=0.010$; $\mathrm{P}$ for $\mathrm{FIN}=0.010$; $\mathrm{P}$ for $\mathrm{IN} 30 \mathrm{~min}=$ 0.001; $P$ for $I N 1 h=0.002$ ). Both HOMA- $\beta$ and Stumvoll formula indexes were positively related to coffee consumption ( $P$ for HOMA- $\beta=0.033$; $P$ for Stumvoll first phase $=0.003$; $P$ for Stumvoll second phase $=0.001$ ). Logistic regression analysis further confirmed that coffee intake was independently associated with higher levels of HOMA- $\beta$ and Stumvoll insulin secretion indexes [OR (95\% Cl) for HOMA- $\beta=2.270$ (1.456-3.538); OR (95\% Cl) for Stumvoll first phase $=2.071$ (1.352-3.173); OR (95\% Cl) for Stumvoll second phase $=1.914$ (1.260-2.906)].

Conclusions: Coffee intake is independently and positively related to pancreatic beta cell function in a large high-risk diabetic Chinese population.
\end{abstract}

Keywords: Coffee, Diabetes, Insulin secretion

\section{Background}

Coffee is one of the most widely used beverages worldwide. In the recent decades, there are lots of studies about the relationship of coffee consumption and a series of diseases, such as cardiovascular disease [1], cerebrovascular disease [2], insomnia [3] and diabetes [4]. Several meta-analyses confirmed that coffee consumption was associated with a lower risk of type 2 diabetes

\footnotetext{
* Correspondence: crwang@sjtu.edu.cn; wpjia@sjtu.edu.cn

${ }^{\dagger}$ Fei Gao and Yinan Zhang contributed equally to this work.

${ }^{4}$ Department of Endocrinology and Metabolism, Shanghai Jiao Tong

University Affiliated Sixth People's Hospital, Shanghai Key Laboratory of

Diabetes, The Metabolic Diseases Biobank, Shanghai, China

Full list of author information is available at the end of the article
}

mellitus [5-7]. As we know, insulin resistance and beta cell function deterioration are the two critical underlying traits of diabetes pathogenesis. However, the connection between coffee and diabetes pathogenesis is still controversial. For instance, a Swedish study implied that coffee intake might involve both improved insulin sensitivity and enhanced insulin response [8]. Nonetheless, some others showed that coffee was associated with insulin sensitivity but not insulin secretion. Arnlov J et al. only found relationship of coffee intake with insulin sensitivity but not with early phase insulin secretion during an oral glucose tolerance test [9]. Thus, it is still significant to

(C) The Author(s). 2018 Open Access This article is distributed under the terms of the Creative Commons Attribution 4.0 International License (http://creativecommons.org/licenses/by/4.0/), which permits unrestricted use, distribution, and 
further investigate the relationship between coffee and the pathogenesis of diabetes.

China is a newly coffee consumption country, more and more people custom to having coffee frequently. Meanwhile, China has large diabetes epidemic.The estimated overall prevalence of total diabetes and of pre-diabetes is 10.9 and $35.7 \%$, respectively [10]. However, the association between coffee consumption and diabetes or the pathogenesis in Chinese population is largely unknown.

In the present study, we aim to evaluate the association of coffee intake and the prevalence of newly diagnosed diabetes as well as diabetic pathogenesis in a large high-risk diabetic Chinese Population from Shanghai High-risk Diabetic Screen project (SHiDS).

\section{Methods}

\section{Study design and population}

The study population was selected from the Shanghai High-Risk Diabetic Screen (SHiDS) project, a large clinic based screening project that was implemented since 2002 . Details on the methodology have previously been reported [11]. In brief, the SHiDS project involves screening of individuals with known risk factors for diabetes. The inclusion criterion of SHiDS study is individuals with at least one of the known risk factors for diabetes including 1) family history of diabetes, 2) being overweight or obese, 3) previously identified impaired fasting glucose or impaired glucose tolerance, 4) history of gestational diabetes, 5) polycystic ovary syndrome, 6) hypertension, 7) dyslipidemia. Previously diagnosed diabetic patients were excluded from the study [11]. For the present study, a total of 1328 individuals over 18 years of age who have the information of coffee intake were enrolled in the study from 2012 to 2016. This study was approved by the Institutional Review Board of Shanghai JiaoTong University Affiliated Sixth People's Hospital in accordance with the principles of the Helsinki Declaration. Informed consent was obtained from each subject before the survey.

\section{Data collection}

Participants arrived at the hospital at 7:30 AM after an $8 \mathrm{~h}$ overnight fasting before the five-point $75 \mathrm{~g}$ oral glucose tolerance test (OGTT). Each participant underwent a physical examination including measurement of height, weight, waist circumference and blood pressure. Body mass index (BMI) was calculated as weight divided by height squared $\left(\mathrm{kg} / \mathrm{m}^{2}\right)$. A standard questionnaire was conducted by trained research staff. Data on the current lifestyle, coffee intake, tea intake, smoking habit, alcohol intake, physical activity, medical record, diabetes family history (first degree relatives only) and educational status were collected for each participant. Venous blood samples were collected at 0, 30, 60, 120 and $180 \mathrm{~min}$ during a standard 75 g-OGTT, and plasma glucose levels were measured using a glucose oxidase method. Glycosylated hemoglobin A1c (HbA1c) was measured by high performance liquid chromatography. Glycated albumin (GA) was measured by the liquid enzymatic assay. Serum insulin levels were measured using a chemical luminescence method.

Diabetes was diagnosed according to the standard criteria by the World Health Organization (WHO) in 1999 [fasting plasma glucose $(\mathrm{FPG}) \geq 7.0 \mathrm{mmol} / \mathrm{L}$ or 2 -h postprandial plasma glucose $(\mathrm{PG} 2 \mathrm{~h}) \geq 11.1 \mathrm{mmol} / \mathrm{L}]$. Hypertension and dislipidemia were defined as self-reported with a validated history diagnosed by doctors. Insulin resistance was assessed by homeostatic model assessment of insulin resistance (HOMA-IR) value [12]. Pancreatic beta cell function was evaluated by homeostatic model assessment of $\beta$-cell function (HOMA- $\beta$ ) value [13], Stumvoll first phase and second phase indexes, respectively [14].

HOMA-IR = fasting serum insulin $(\mathrm{FIN})(\mathrm{mU} / \mathrm{L}) \times$ FPG $(\mathrm{mmol} / \mathrm{L}) / 22.5[12]$

HOMA- $\beta=20 \times$ FIN $(\mathrm{mU} / \mathrm{L}) /[\mathrm{FPG}(\mathrm{mmol} / \mathrm{L})-3.5]$ [13]

Stumvoll1st phase $=2503+38.856 \times$ FIN $(\mathrm{mU} / \mathrm{L}) \quad-$ $126.5 \times \mathrm{PG} 2 \mathrm{~h}(\mathrm{mmol} / \mathrm{l})+5.724 \times 2$-h postprandial serum insulin (IN2h) $(\mathrm{mU} / \mathrm{L})-239.3 \times \mathrm{FPG}(\mathrm{mmol} / \mathrm{l})$ [14] .

Stumvoll 2nd phase $=393+6.978 \times$ FIN $(\mathrm{mU} / \mathrm{L}) \quad-$ $40.72 \times \mathrm{PG} 2 \mathrm{~h}(\mathrm{mmol} / \mathrm{l})+1.878 \times \mathrm{IN} 2 \mathrm{~h}(\mathrm{mU} / \mathrm{L})[14]$

\section{Statistical analyses}

Statistical analyses were performed using SPSS 20.0 (SPSS, Inc., Chicago, IL). FPG, 30-min postprandial plasma glucose (PG30min), 1-h postprandial plasma glucose (PG1h), PG2h, 3-h postprandial plasma glucose (PG3h), FIN, 30-min postprandial serum insulin (IN30min), 1-h postprandial serum insulin (IN1h), IN2h, 3-h postprandial serum insulin (IN3h), HbA1c, GA and HOMA-IR were logarithmic transformed to obtain approximate normal distribution before statistical analysis. Participant characteristics were tested for differences across coffee intake categories. Variables with approximately normal distribution were presented as means \pm standard deviation while those with skew distribution were shown as median [interquartile range (IQR)] and classified variable as frequencies and percentages. Continuous variables were compared by $\mathrm{t}$-test while classified variable by chi-square test. The associations of coffee intake with different variables were analyzed by covariance. Logistic regression was used to model associations between coffee consumption and insulin secretion indexes, taking account of potential confounders. All reported $P$ values were two-tailed and $P<0.05$ were considered statistically significant.

\section{Result}

Clinical characteristics of the participants stratified by coffee consumption

The demographic and clinical characteristics of the participants are presented in Table 1. After statistical analysis by 
Table 1 Clinical characteristics of the participants stratified by coffee consumption

\begin{tabular}{|c|c|c|c|c|}
\hline & Non-coffee $(n=818)$ & Coffee $(n=510)$ & Total $(n=1328)$ & $P$ \\
\hline Male (n, \%) & $330(40.34 \%)$ & $210(41.18 \%)$ & $540(40.66 \%)$ & 0.763 \\
\hline Age, years (mean, SD) & $52.80(14.22)$ & $48.34(14.49)$ & $51.08(14.47)$ & $<0.001$ \\
\hline Weight, kg (mean, SD) & $65.07(12.60)$ & $69.99(14.24)$ & $66.95(13.46)$ & $<0.001$ \\
\hline BMI, kg/m² (mean, SD) & $23.96(3.65)$ & $25.18(4.08)$ & $24.43(3.87)$ & $<0.001$ \\
\hline Waist, cm (mean, SD) & $86.46(10.19)$ & $88.79(11.07)$ & $87.35(10.59)$ & $<0.001$ \\
\hline Fat, \% (mean, SD) & $28.37(7.70)$ & $29.81(8.25)$ & $28.91(7.94)$ & 0.004 \\
\hline SBP, mmHg (mean, SD) & $130.39(18.17)$ & $130.23(17.41)$ & $130.33(17.88)$ & 0.880 \\
\hline DBP, mmHg (mean, SD) & $79.02(10.93)$ & $79.20(11.33)$ & 79.09 (11.09) & 0.776 \\
\hline Diabetic family history (n, \%) & 351 (44.94\%) & 249 (50.82\%) & 1271 (47.21\%) & 0.041 \\
\hline Hypertension (n, \%) & $279(34.15 \%)$ & $160(31.37 \%)$ & 1327 (33.08\%) & 0.296 \\
\hline Dislipidemia (n, \%) & 249 (30.48\%) & 168 (33.01\%) & $1326(31.45 \%)$ & 0.335 \\
\hline Higher Education (n, \%) & $370(45.23 \%)$ & $312(61.18 \%)$ & $1328(51.36 \%)$ & $<0.001$ \\
\hline Current smoke (n, \%) & $104(12.73 \%)$ & $91(17.88 \%)$ & $1326(14.71 \%)$ & 0.010 \\
\hline Current alcohol intake (n, \%) & 115/703 (14.06\%) & 107/402 (21.02\%) & $222(16.73 \%)$ & 0.001 \\
\hline Tea consumption (n, \%) & $293(35.91 \%)$ & $321(62.94 \%)$ & $1326(46.30 \%)$ & $<0.001$ \\
\hline Frequent physical activity (n, \%) & 629 (76.99\%) & 385 (75.49\%) & 1327 (76.41\%) & 0.532 \\
\hline FPG, mmol/L (median, IQR) & $6.15(5.46-7.06)$ & $6.04(5.36-7.14)$ & $6.11(5.42-7.08)$ & 0.259 \\
\hline PG30min, mmol/L (median, IQR) & $10.67(9.09-12.38)$ & $10.47(9.05-12.27)$ & $10.60(9.07-12.35)$ & 0.188 \\
\hline PG1h, mmol/L (median, IQR) & $12.29(9.79-15.25)$ & $12.19(9.43-14.97)$ & $12.25(9.68-15.14)$ & 0.148 \\
\hline PG2h, mmol/L (median, IQR) & $10.02(7.36-14.31)$ & $9.53(7.09-13.47)$ & $9.85(7.28-14.06)$ & 0.024 \\
\hline PG3h, mmol/L (median, IQR) & $6.69(4.84-9.62)$ & $6.30(4.55-8.97)$ & $6.45(4.74-9.35)$ & 0.016 \\
\hline FIN, ulU/mL (median, IQR) & $8.43(5.75-12.61)$ & $10.10(6.94-16.22)$ & $9.08(6.02-13.81)$ & $<0.001$ \\
\hline IN30min, ulU/mL (median, IQR) & $44.05(27.52-69.97)$ & 58.09 (34.50-93.70) & $48.21(28.82-80.23)$ & $<0.001$ \\
\hline IN1h, ulU/mL (median, IQR) & $64.58(40.18-101.45)$ & 77.03 (50.30-121.83) & $69.20(44.25-107.50)$ & $<0.001$ \\
\hline IN2h, ulU/mL (median, IQR) & $69.91(45.83-115.70)$ & $81.54(51.53-128.38)$ & 74.75 (48.05-119.05) & 0.002 \\
\hline IN3h, ulU/mL (median, IQR) & $32.22(17.34-57.65)$ & $34.29(18.23-62.04)$ & $32.75(17.64-58.38)$ & 0.237 \\
\hline HbA1c, \% (median, IQR) & $6.00(5.60-6.50)$ & $5.90(5.50-6.60)$ & $5.90(5.60-6.50)$ & 0.855 \\
\hline GA, \% (median, IQR) & $14.30(12.80-16.60)$ & $13.85(12.40-16.70)$ & $14.10(12.70-16.68)$ & 0.263 \\
\hline Newly diagnosed diabetes (n, \%) & 381 (46.58\%) & $213(41.76 \%)$ & $594(44.73 \%)$ & 0.037 \\
\hline Impaired glucose regulation (n, \%) & 237 (28.97\%) & $148(29.02 \%)$ & 385 (28.99\%) & 0.241 \\
\hline HOMA- $\beta$ (mean, SD) & $84.30(89.77)$ & $104.65(103.29)$ & $92.11(95.66)$ & $<0.001$ \\
\hline HOMA-IR (median, IQR) & $2.37(1.49-3.71)$ & $2.80(1.75-4.61)$ & $2.52(1.60-4.04)$ & $<0.001$ \\
\hline Stumvoll first phase (mean, SD) & 459.97 (1096.52) & 717.67 (1060.32) & 558.85 (1089.60) & $<0.001$ \\
\hline Stumvoll second phase (mean, SD) & $179.32(248.52)$ & $242.04(246.55)$ & $203.33(249.54)$ & $<0.001$ \\
\hline
\end{tabular}

Continuous variables were compared by t-test while classified variable by chi-square test

Variables with approximately normal distribution were presented as means \pm standard deviation while those with skew distribution were shown as median (inter quartile range) and classified variable as frequencies and percentages

Abbreviations: $S D$ standard deviation, $I Q R$ inter quartile range, $B M I$ body mass index, $S B P$ systolic pressure, $D B P$ diastolic pressure, $F P G$ fasting plasma glucose, PG30min 30-min postprandial plasma glucose, $P G 1 \mathrm{~h}$ 1-h postprandial plasma glucose, $P G 2 \mathrm{~h}$ 2-h postprandial plasma glucose, $P G 3 \mathrm{~h}$ 3-h postprandial plasma glucose, HbA1c glycosylated hemoglobin A1c, GA glycated albumin, FIN fasting serum insulin, IN30min 30-min postprandial serum insulin, IN1 $\mathrm{h}$ 1-h postprandial serum insulin, IN2h 2-h postprandial serum insulin, IN3h 3-h postprandial serum insulin, HOMA-IR homeostatic model assessment of insulin resistance, HOMA- $\beta$, homeostatic model assessment of $\beta$-cell function

t-test, it showed that coffee consumed population was relatively younger and fatter than non-coffee participants ( $P$ for age $<0.001 ; P$ for BMI $<0.001 ; P$ for waist $<0.001 ; P$ for fat $\%=0.004)$. Besides, coffee consumed group had higher education level $(P<0.001)$, current smoke percentage $(P=0.010)$, current alcohol intake percentage $(P=0.001)$, tea consumption percentage $(P<0.001)$ and higher percentage of diabetes family history $(P=0.041)$. The prevalence of hypertension and dyslipidemia were not different between these two groups. 
In terms of glucose metabolism, the coffee intake group had lower 2-h and 3-h plasma glucose levels during OGTT ( $P$ for PG2h $=0.024 ; P$ for PG3h $=0.016$ ). However, the plasma glucose levels at fasting, 30-min, 1 -h were not different between these two populations, so as the HbA1c level and GA level. According to the chi-square test, coffee intake group had lower prevalence of newly diagnosed diabetes $(P=0.037)$.

In terms of insulin levels, coffee consumption group had higher insulin levels at fasting, 30-min, 1-h and 2-h during OGTT $(P$ for FIN $<0.001 ; P$ for IN30min $<$ 0.001; $P$ for $\mathrm{IN} 1 \mathrm{~h}<0.001 ; P$ for $\mathrm{IN} 2 \mathrm{~h}=0.002)$, but serum insulin level at 3-h was not different from the non-coffee intake group. Pancreatic beta cell function and insulin resistance were further estimated by HOMA and Stumvoll indexes. It implied that coffee intake individuals had higher insulin secretion level $(P$ for
HOMA- $\beta<0.001 ; P$ for Stumvoll first phase $<0.001 ; P$ for Stumvoll second phase $<0.001)$ and lower insulin sensitivity $(P$ for HOMA-IR $<0.001)$.

\section{Coffee consumption and glucose metabolism, insulin secretion and insulin sensitivity}

The relationships of coffee intake and glucose metabolism, insulin secretion and insulin sensitivity were further analyzed by different adjusted models (Table 2). First of all, coffee consumption group had lower plasma glucose levels at 2-h and 3-h and higher insulin levels at fasting, 30-min and 1-h during OGTT after adjustment with age, fat\%, BMI, waist, tea intake, smoking habit, alcohol intake, diabetes family history and educational status $(P$ for $\mathrm{PG} 2 \mathrm{~h}=0.002 ; P$ for $\mathrm{PG} 3 \mathrm{~h}=0.010 ; P$ for $\mathrm{FIN}=0.010$; $P$ for IN30min $=0.001$; $P$ for IN1h $=0.002$ ). Nevertheless, the difference of the prevalence of newly diagnosed

Table 2 Adjusted Mean (SD) values for metabolic measures according to the relationship with coffee consumption

\begin{tabular}{|c|c|c|c|c|c|c|c|}
\hline \multirow[t]{2}{*}{ lgPG2h } & \multicolumn{7}{|c|}{ lgPG3h } \\
\hline & Non-coffee & Coffee & $P^{*}$ & & Non-coffee & Coffee & $p^{*}$ \\
\hline Model 1 & $1.01 \pm 0.18$ & $0.98 \pm 0.19$ & 0.477 & Model 1 & $0.84 \pm 0.21$ & $0.81 \pm 0.21$ & 0.122 \\
\hline Model 2 & $1.01 \pm 0.18$ & $0.99 \pm 0.19$ & 0.020 & Model 2 & $0.85 \pm 0.21$ & $0.82 \pm 0.21$ & 0.021 \\
\hline \multirow[t]{3}{*}{ Model 3} & $1.02 \pm 0.18$ & $0.99 \pm 0.19$ & 0.002 & Model 3 & $0.85 \pm 0.21$ & $0.82 \pm 0.21$ & 0.010 \\
\hline & \multicolumn{2}{|c|}{$\lg F I N$} & & \multicolumn{4}{|c|}{ IgIN30min } \\
\hline & Non-coffee & Coffee & $p^{*}$ & & Non-coffee & Coffee & $p^{*}$ \\
\hline Model 1 & $0.92 \pm 0.29$ & $1.01 \pm 0.29$ & $<0.001$ & Model 1 & $1.64 \pm 0.34$ & $1.74 \pm 0.36$ & $<0.001$ \\
\hline Model 2 & $0.92 \pm 0.30$ & $1.01 \pm 0.29$ & 0.012 & Model 2 & $1.64 \pm 0.34$ & $1.75 \pm 0.35$ & 0.002 \\
\hline Model 3 & $0.92 \pm 0.29$ & $1.01 \pm 0.29$ & 0.010 & Model 3 & $1.64 \pm 0.34$ & $1.74 \pm 0.35$ & 0.001 \\
\hline \multirow[t]{2}{*}{ lgIN1h } & & & & lgIN2h & & & \\
\hline & Non-coffee & Coffee & $p^{*}$ & & Non-coffee & Coffee & $p^{*}$ \\
\hline Model 1 & $1.80 \pm 0.31$ & $1.87 \pm 0.32$ & $<0.001$ & Model 1 & $1.85 \pm 0.31$ & $1.90 \pm 0.31$ & 0.004 \\
\hline Model 2 & $1.79 \pm 0.30$ & $1.87 \pm 0.31$ & 0.014 & Model 2 & $1.85 \pm 0.31$ & $1.90 \pm 0.30$ & 0.287 \\
\hline Model 3 & $1.79 \pm 0.30$ & $1.87 \pm 0.31$ & 0.002 & Model 3 & $1.85 \pm 0.31$ & $1.90 \pm 0.30$ & 0.120 \\
\hline \multicolumn{4}{|c|}{ IgHOMA-IR } & \multicolumn{4}{|c|}{ HOMA- $\beta$} \\
\hline & Non-coffee & Coffee & $P^{*}$ & & Non-coffee & Coffee & $p^{*}$ \\
\hline Model 1 & $0.37 \pm 0.32$ & $0.44 \pm 0.33$ & $<0.001$ & Model 1 & $84.30 \pm 89.77$ & $104.65 \pm 103.29$ & 0.025 \\
\hline Model 2 & $0.37 \pm 0.33$ & $0.45 \pm 0.32$ & 0.053 & Model 2 & $80.59 \pm 85.37$ & $104.70 \pm 96.93$ & 0.090 \\
\hline Model 3 & $0.37 \pm 0.32$ & $0.45 \pm 0.32$ & 0.085 & Model 3 & $78.06 \pm 70.61$ & $99.44 \pm 74.48$ & 0.033 \\
\hline \multicolumn{4}{|c|}{ Stumvoll first phase } & \multicolumn{4}{|c|}{ Stumvoll second phase } \\
\hline & Non-coffee & Coffee & $p^{*}$ & & Non-coffee & Coffee & $p^{*}$ \\
\hline Model 1 & $459.97 \pm 1096.52$ & $717.67 \pm 1060.32$ & 0.005 & Model 1 & $179.32 \pm 248.52$ & $242.04 \pm 246.55$ & 0.002 \\
\hline Model 2 & $415.95 \pm 1102.94$ & $681.45 \pm 1041.09$ & 0.045 & Model 2 & $170.08 \pm 249.30$ & $236.52 \pm 236.91$ & 0.020 \\
\hline Model 3 & $392.53 \pm 1101.89$ & $659.70 \pm 1029.83$ & 0.003 & Model 3 & $166.15 \pm 249.01$ & $232.98 \pm 233.96$ & 0.001 \\
\hline
\end{tabular}

Values presented as Mean \pm SD. PG2h, PG3h, FIN, IN30min, IN1h, IN2h and HOMA-IR were logarithmic transformed

* $P$-value for the test of any association between coffee consumption and the outcome of interest

Model 1: adjusted for age

Model 2: Model $1+$ BMI, Fat\%, Waist

Model 3: Model $2+$ education status, smoke status, alcohol intake, tea consumption, diabetes family history

Abbreviations: $S D$ standard deviation, $B M I$ body mass index, $P G 2 h$ 2-h postprandial plasma glucose, $P G 3 h$ 3-h postprandial plasma glucose, FIN fasting serum insulin, IN30min 30-min postprandial serum insulin, IN $1 \mathrm{~h}$ 1-h postprandial serum insulin, IN2h 2-h postprandial serum insulin, HOMA-IR homeostatic model assessment of insulin resistance, HOMA- $\beta$ homeostatic model assessment of $\beta$-cell function 
diabetes between these two groups was not significant after adjusted with age $(P=0.718)$. Secondly, a relatively strong association was found between coffee consumption and insulin secretion after adjusted with variety confounders mentioned above that might interfere with the relationship of coffee intake and pancreatic beta cell function. Both HOMA- $\beta$ and Stumvoll formula indexes were positively related to coffee consumption ( $P$ for HOMA- $\beta=0.033 ; P$ for Stumvoll first phase $=0.003 ; P$ for Stumvoll second phase $=0.001$ ). However, insulin sensitivity index was not statistically different between coffee consumed population and non-coffee consumed population after adjustment.

\section{Logistic regression analysis about the relationship of insulin secretion and coffee consumption}

Logistic regression was conducted to further analysis the relationship of coffee intake and insulin secretion (Table 3). Insulin secretion indexes were expressed in quartiles and were taken as dependent variable. Coffee intake and the other parameters (age, gender distribution, BMI, fat\%, waist, education status, smoke status, alcohol intake, tea consumption, diabetes family history) were taken as independent variables. It showed that coffee consumption was significantly and positively associated with insulin secretion. After adjusted with the confounders (model 1, model 2 and model 3), Logistic regression analysis further confirmed that coffee consumers had higher levels of HOMA- $\beta$ and Stumvoll

Table 3 Odds ratios ( $95 \%$ Cl) for the association between coffee consumption and insulin secretion indexes

\begin{tabular}{clcc}
\hline & HOMA- $\beta$ (the highest quartiles) & \\
coffee consumption & OR & $95 \% \mathrm{Cl}$ & $P^{*}$ \\
Model 1 & 2.240 & $1.598-3.140$ & $<0.001$ \\
Model 2 & 2.048 & $1.359-3.086$ & 0.001 \\
Model 3 & 2.262 & $1.451-3.527$ & $<0.001$ \\
& Stumvoll first phase (the highest quartiles) \\
coffee consumption & OR & $95 \% \mathrm{Cl}$ & $P^{*}$ \\
Model 1 & 1.818 & $1.297-2.549$ & 0.001 \\
Model 2 & 1.807 & $1.222-2.672$ & 0.003 \\
Model 3 & 2.041 & $1.331-3.131$ & 0.001 \\
& Stumvoll second phase (the highest quartiles) \\
coffee consumption & OR & $95 \% \mathrm{Cl}$ & $P^{*}$ \\
Model 1 & 1.730 & $1.238-2.419$ & 0.001 \\
Model 2 & 1.762 & $1.196-2.595$ & 0.004 \\
Model 3 & 1.890 & $1.243-2.874$ & 0.003 \\
\hline
\end{tabular}

*P-value for the test of any association between coffee consumption and the outcome of interest

Model 1: adjusted for age, gender distribution

Model 2: Model $1+$ BMI, Fat\%, Waist

Model 3: Model $2+$ education status, smoke status, alcohol intake, tea

consumption, diabetes family history insulin secretion indexes (model 3: odds ratio (OR) [95\% confidence interval $(\mathrm{CI})]$ for HOMA- $\beta=2.262(1.451-$ 3.527); OR (95\% CI) for Stumvoll first phase $=2.041$ (1.331-3.131); OR (95\% CI) for Stumvoll second phase $=1.890(1.243-2.874)\}$. Thus, participant consumed coffee might have better insulin secretion function, including the basic insulin secretion and the two phases of insulin secretion during OGTT.

\section{Discussion}

As far as we know, the present study was the first investigation about relationship between coffee intake and diabetes as well as its pathogenesis in a large high-risk diabetic Chinese population. It showed that coffee intake was positively associated with fasting and the first hour insulin levels and inversely associated with second and third hour plasma glucose levels during the OGTT. In addition, Logistic analysis revealed that coffee consumption was independently and positively related to a series of insulin secretion indexes, including HOMA- $\beta$, Stumvoll first and second phase insulin secretion indexes. The HOMA calculation is derived from a computer solved model that assumes relationships between fasting plasma glucose and insulin concentration [13]. HOMA- $\beta$ evaluated the basic insulin secretion function [15]. Stumvoll 1st and 2nd phase insulin secretion indexes are simple demographic parameters and they can evaluate insulin release accuracy [16]. The significance of the first phase insulin secretion might reflect the existence of a compartment of readily releasable insulin within the beta cell or a transientrise and fall of a metabolic signal for insulin secretion [17, 18]. The second phase of insulin secretion is directly related to the level of glucose elevation [18]. Thus, the present study implied that coffee consumers not only had better basal pancreatic beta cell function, but also had superior postprandial beta cell function.

Our findings were consistent with some previous investigations. The results from Agardh et al. showed that in those with type 2 diabetes high coffee consumption was inversely associated with decreased beta cell function which was evaluated by HOMA- $\beta$ [8]. It suggested an effect of coffee on beta-cell function and there was a tendency of improved insulin response in those with high coffee consumption [8]. The results from Wu TY et al. provided support for the potential benefit of chronic coffee consumption on insulin secretion and possibly diabetes $[19,20]$. However, there are some inconsistencies in the effect of coffee on insulin secretion. In a cross-sectional analysis of Japanese population, higher coffee consumption was not associated with insulin secretion, as evaluated through the HOMA [21]. Another cross-sectional study from the Uppsala Longitudinal Study of Adult Men showed that there was no 
association between coffee consumption and early insulin response during an OGTT [9]. The differences in race and population selection criteria might cause different results of relationship between coffee intake and insulin secretion.

The mechanisms that coffee may have beneficial effects on pancreatic beta cell function are still unclear. Coffee contains a lot of components. Caffeine is the major one which is already known to enhance insulin secretion $[8,22]$. It has been proved that insulin concentration tended to be higher in the first $30 \mathrm{~min}$ after caffeinated coffee consumption compared with that of decaffeinated coffee or water [23]. Another study revealed that insulin was significantly higher after caffeine coffee than after water during the first hour of the OGTT [24]. Other major components of coffee including magnesium, chlorogenic acid and various other micronutrients could also be involved in insulin secretion [25]. Chlorogenic acid is another major ingredient in coffee. There is evidence that chlorogenic acid might stimulate glucagon-like peptide 1 (GLP-1) production which is one of the gastrointestinal hormones and is known to have an effect on beta cell function that amplifies glucose-dependent insulin secretion $[23,26]$. On the other hand, magnesium has also been shown to improve $\beta$-cell function. Previous study found that increase in erythrocyte magnesium significantly and positively correlated with the increase in both insulin secretion and action [27]. Thus, more and deeper investigations are necessary to reveal the mechanisms that coffee is benefit for insulin secretion and glucose homeostasis.

There are some strengths of our study. First of all, each diabetic patient was newly diagnosed by astandard $75 \mathrm{~g}$-OGTT. Thus, the effect of anti-diabetic medication on the relationship of coffee intake and hyperglycemia can be excluded. We also tested the 5-point blood glucose and insulin levels during the OGTT, which made it possible for us to entirely investigate the dynamic fluctuations in insulin secretion after glucose load. Secondly, to our knowledge, there were few studies on coffee intake and hyperglycemia in Chinese population. The population of China accounts for one fifth of the world's population and China is becoming an emerging coffee consumer country. According to the 2017-2021 China coffee industry development prospect forecast and investment analysis report, coffee consumption is growing by 15 to $20 \%$ a year in China [28]. Thus, it is especially important to evaluate the relationship of coffee intake with glucose metabolism in Chinese population. Our study extended the apparent protective effect of coffee on insulin secretion and glucose metabolism.

Except for the major results of the present study, we also found that coffee consumed group had higher current smoke percentage, current alcohol intake percentage and tea consumption percentage. These results were consistent with the previous studies $[8,19,25,29]$. It is well-known that alcohol, tobacco, tea and coffee can cause varying degrees of addiction or dependence [3032]. Our findings implied that there might be some concentration effect on dependency or addictive substances, i.e. coffee, tea, alcohol and tobacco, which needs further study.

\section{Conclusions}

In the present study, we provide solid and strong evidence that coffee intake is independently and positively related to pancreatic beta cell function in a large high-risk diabetic Chinese population. Habitual coffee consumption population might have better pancreatic beta cell function in Chinese population.

\section{Abbreviations}

BMI: Body mass index; Cl: Confidence interval; DBP: Diastolic pressure; FIN: Fasting serum insulin; FPG: Fasting plasma glucose; GA: Glycated albumin; GLP-1: Glucagon-like peptide 1; HbA1c: Glycosylated

hemoglobin A1c; HOMA-IR: Homeostatic model assessment of insulin resistance; HOMA- $\beta$ : Homeostatic model assessment of $\beta$-cell function; IN1h: 1-h postprandial serum insulin; IN2h: 2-h postprandial serum insulin; IN30min: 30-min postprandial serum insulin; IN3h: 3-h postprandial serum insulin; IQR: Inter quartile range; OGTT: Oral glucose tolerance test; OR: odds ratio; PG1h: 1-h postprandial plasma glucose; PG2h: 2-h postprandial plasma glucose; PG30min: 30-min postprandial plasma glucose; PG3h: 3-h postprandial plasma glucose; SBP: Systolic pressure; SD: Standard deviation; SHiDS: Shanghai High-Risk Diabetic Screen; WHO: World Health Organization

\section{Acknowledgements}

The authors thank The Metabolic Diseases Biobank of Shanghai Jiao Tong University Affiliated Sixth People's Hospital for collecting data and offering help.

\section{Funding}

This work was financially supported by funding from the National Key Research and Development Program of China (Grant No.2017YFC0906903), the National Natural Science Foundation of China (Grant No.81570808, No.81770883), a Municipal Human Resources Development Program for Outstanding Leaders in Medical Disciplines in Shanghai (Grant No.2017BR045) and National Human Genetic Resources Sharing Service Platform (Grant No. YCZYPT [2017]02).

\section{Availability of data and materials}

The datasets used and analyzed during the current study are available from the corresponding author on reasonable request.

\section{Authors' contributions}

FG made substantial contributions to design the study, organized and analyzed the data and was the major contributor in writing the manuscript. YZ made contributions to design the study and analyzed the data. FG and YZ contributed equally to this work. SG contributed to design and helped to provide knowledge of clinical nutrition. $\mathrm{HL}, \mathrm{RC}, \mathrm{PF}$ and $\mathrm{YS}$ were responsible for the subject follow-up and questionnaires as well as data collection. CW was the corresponding author and directed the study design, data analysis as well as manuscript writing. WJ was also the corresponding author and directed the study design as well as data analysis. All authors read and approved the final manuscript.

Authors' information

Not applicable.

\section{Ethics approval and consent to participate}

This study was approved by the Institutional Review Board of Shanghai Jiao Tong University Affiliated Sixth People's Hospital in accordance with the 
principles of the Helsinki Declaration. Informed consent was obtained from each subject before the survey.

\section{Consent for publication \\ Not applicable.}

\section{Competing interests}

The authors declare that they have no competing interests.

\section{Publisher's Note}

Springer Nature remains neutral with regard to jurisdictional claims in published maps and institutional affiliations.

\section{Author details}

'Department of Endocrinology and Metabolism, Shanghai Jiao Tong University Affiliated Sixth People's Hospital, Shanghai Clinical Medical Center of Diabetes, Shanghai Key Clinical Center of Diabetes, Shanghai Diabetes Institute, Shanghai Key Laboratory of Diabetes, Shanghai, China. ${ }^{2}$ Shanghai Jiao Tong University Affiliated Sixth People's Hospital, Shanghai Key Laboratory of Diabetes, The Metabolic Diseases Biobank, Center for Translational Medicine, Shanghai, China. ${ }^{3}$ Department of Clinical Nutrition, Shanghai Jiao Tong University Affiliated Sixth People's Hospital, Shanghai, China. ${ }^{4}$ Department of Endocrinology and Metabolism, Shanghai Jiao Tong University Affiliated Sixth People's Hospital, Shanghai Key Laboratory of Diabetes, The Metabolic Diseases Biobank, Shanghai, China.

Received: 7 September 2018 Accepted: 13 November 2018 Published online: 27 November 2018

\section{References}

1. Guessous I, Pruijm M, Ponte B, Ackermann D, Ehret G, Ansermot N, Vuistiner $P$, Staessen J, Gu Y, Paccaud F, Mohaupt M, Vogt B, Pechère-Bertschi A, Martin PY, Burnier M, Eap CB, Bochud M. Associations of ambulatory blood pressure with urinary caffeine and caffeine metabolite excretions. Hypertension. 2015;65(3):691-6.

2. Sakamaki T, Hara M, Kayaba K, Kotani K, Ishikawa S. Coffee consumption and incidence of subarachnoid hemorrhage: the Jichi medical school cohort study. J Epidemiol. 2016;26(2):71-5.

3. Watson EJ, Coates AM, Kohler M, Banks S. Caffeine consumption and sleep quality in Australian adults. Nutrients. 2016;8(8) pii: E479.

4. Doo T, Morimoto Y, Steinbrecher A, Kolonel LN, Maskarinec G. Coffee intake and risk of type 2 diabetes: the multiethnic cohort. Public Health Nutr. 2014; 17(6):1328-36.

5. Huxley R, Lee CM, Barzi F, Timmermeister L, Czernichow S, Perkovic V, Grobbee DE, Batty D, Woodward M. Coffee, decaffeinated coffee, and tea consumption in relation to incident type 2 diabetes mellitus: a systematic review with meta-analysis. Arch Intern Med. 2009;169(22):2053-63.

6. Ding M, Bhupathiraju SN, Chen M, van Dam RM, Hu FB. Caffeinated and decaffeinated coffee consumption and risk of type 2 diabetes: a systematic review and a dose-response meta-analysis. Diabetes Care. 2014;37(2):569-86.

7. Jiang $X$, Zhang $D$, Jiang $W$. Coffee and caffeine intake and incidence of type 2 diabetes mellitus: a meta-analysis of prospective studies. Eur J Nutr. 2014; 53(1):25-38

8. Agardh EE, Carlsson S, Ahlbom A, Efendic S, Grill V, Hammar N, Hilding A, Ostenson CG. Coffee consumption,type 2 diabetes and impaired glucose tolerance in Swedish men and women. J Intern Med. 2004;255(6):645-52.

9. Arnlov J, Vessby B, Riserus U. Coffee consumption and insulin sensitivity. JAMA. 2004;291(10):1199-201.

10. Wang L, Gao P, Zhang M, Huang Z, Zhang D, Deng Q, Li Y, Zhao Z, Qin X, Jin D, Zhou M, Tang X, Hu Y, Wang L. Prevalence and ethnic pattern of diabetes and prediabetes in China in 2013. JAMA. 2017;317(24):2515-23.

11. Wang C, Zhang Y, Zhang L, Hou X, Lu H, Shen Y, Chen R, Fang P, Yu H, Li M, Zhang F, Chen H, Yu H, Zhou J, Liu F, Bao Y, Jia W. Prevalence of type 2 diabetes among high-risk adults in Shanghai from 2002 to 2012. PLoS One. 2014;9(7):e102926.

12. Gutt M, Davis CL, Spitzer SB, Llabre MM, Kumar M, Czarnecki EM, Schneiderman N, Skyler JS, Marks JB. Validation of the insulin sensitivity index (ISI $(0,120))$ : comparison with other measures. Diabetes Res Clin Pract. 2000;47(3):177-84.

13. Matthews DR, Hosker JP, Rudenski AS, Naylor BA, Treacher DF, Turner RC. Homeostasis model assessment: insulin resistance and beta-cell function from fasting plasma glucose and insulin concentrations in man. Diabetologia. 1985;28(7):412-9.

14. Stumvoll M, Van Haeften T, Fritsche A, Gerich J. Oral glucose tolerance test indexes for insulin sensitivity and secretion based on various availabilities of sampling times. Diabetes Care. 2001;24(4):796-7.

15. Zheng S, Zhou H, Han T, Li Y, Zhang Y, Liu W, Hu Y. Clinical characteristics and beta cell function in Chinese patients with newly diagnosed type 2 diabetes mellitus with different levels of serum triglyceride. BMC Endocr Disord. 2015;15:21.

16. Stumvoll M, Mitrakou A, Pimenta W, Jenssen T, Yki-Järvinen H, Van Haeften T, Renn W, Gerich J. Use of the oral glucose tolerance test to assess insulin release and insulin sensitivity. Diabetes Care. 2000;23(3):295-301.

17. Grodsky GM. A threshold distribution hypothesis for packet storage of insulin and its mathematical modeling. J Clin Invest. 1972;51(8):2047-59.

18. Buse JB, Polonsky KS, Burant CF. Type 2 diabetes mellitus. In: Kronenberg HM, Melmed S, Polonsky KS, Larsen PR, editors. Willams textbook of endocrinology, 11/E. Elsevier; 2008. P. 1348.

19. Wu T, Willett WC, Hankinson SE, Giovannucci E. Caffeinated coffee, decaffeinated coffee, and caffeine in relation to plasma C-peptide levels, a marker of insulin secretion, in U.S. women. Diabetes Care. 2005;28(6):1390-6.

20. Salazar-Martinez E, Willett WC, AscherioA MJE, Leitzmann MF, StampferMJ HFB. Coffee consumption and risk for type 2 diabetes mellitus. Ann Intern Med. 2004;140(1):1-8.

21. Pham NM, Nanri A, Kochi T, Kuwahara K, Tsuruoka H, Kurotani K, Akter S, Kabe I, Sato M, Hayabuchi H, Mizoue T. Coffee and green tea consumption is associated with insulin resistance in Japanese adults. Metabolism. 2014;63(3):400-8.

22. Shi CL. Effects of caffeine and acetylcholine on glucose-stimulated insulin release from islet transplants in mice. Cell Transplant. 1997;6(1):33-7.

23. Johnston KL, Clifford MN, Morgan LM. Coffee acutely modifies gastrointestinal hormone secretion and glucose tolerance in humans: glycemic effects of chlorogenic acid and caffeine. Am J Clin Nutr. 2003;78(4):728-33.

24. Greenberg JA, Owen DR, Geliebter A. Decaffeinated coffee and glucose metabolism in young men. Diabetes Care. 2010;33(2):278-80.

25. Yarmolinsky J, Mueller NT, Duncan BB, Bisi Molina Mdel C, Goulart AC, Schmidt MI. Coffee consumption, newly diagnosed diabetes, and other alterations in glucose homeostasis: a cross-sectional analysis of the longitudinal study of adult health (ELSA-Brasil). PLoS One. 2015;10(5):e0126469.

26. McCarty MF. A chlorogenic acid-induced increase in GLP-1 production may mediate the impactof heavy coffee consumption on diabetes risk. Med Hypotheses. 2005;64(4):848-53.

27. Paolisso G, Sgambato S, Gambardella A, Pizza G, Tesauro P, Varricchio M, D'Onofrio F. Daily magnesium supplements improve glucose handling in elderly subjects. Am J Clin Nutr. 1992;55(6):1161-7.

28. 2017-2021 China coffee industry development prospect forecast and investment analysis report. Beijing Zhong Hong Jing Liang Information Consulting Co., Ltd. 2016, https:/www.cn-bigdata.cn/. Accessed 21 March 2018.

29. Loopstra-Masters RC, Liese AD, Haffner SM, Wagenknecht LE, Hanley AJ. Associations between the intake of caffeinated and decaffeinated coffee and measures of insulin sensitivity and betacell function. Diabetologia. 2011; 54(2):320-8.

30. Luciano M, Kirk KM, Heath AC, Martin NG. The genetics of tea and coffee drinking and preference for source of caffeine in a large community sample of Australian twins. Addiction. 2005;100(10):1510-7.

31. Ferraguti G, Pascale E, Lucarelli M. Alcohol addiction: a molecular biology perspective. Curr Med Chem. 2015;22(6):670-84.

32. Scragg R, Difranza JR, Youdan B, Laugesen M, Glover M. Ethnicity and socioeconomic status as risk factors for rapid onset of tobacco addiction. Aust N Z J Public Health. 2014;38(2):194-5.

\section{Ready to submit your research? Choose BMC and benefit from:}

- fast, convenient online submission

- thorough peer review by experienced researchers in your field

- rapid publication on acceptance

- support for research data, including large and complex data types

- gold Open Access which fosters wider collaboration and increased citations

- maximum visibility for your research: over $100 \mathrm{M}$ website views per year

At $\mathrm{BMC}$, research is always in progress.

Learn more biomedcentral.com/submissions 